\title{
Conflict resolution styles and their relation to conflict type, individual differences, and formative influences
}

\author{
JOHN STEVEN GRACE and RICHARD JACKSON HARRIS \\ Kansas State University, Manhattan, Kansas
}

\begin{abstract}
Conflict-resolution styles and formative influences behind them were explored in a study where 207 subjects read conflict scenarios and rated the desirability of various resolutions. Their own attitudes about conflict and a self-rating of the importance of various formative influences in the development of these attitudes were also assessed. Results showed that resolutions to personal conflicts were rated higher than those to international or organizational conflicts. Parents, family, and friends were rated the most important formative influences on the development of styles of conflict resolution.
\end{abstract}

Studies of conflict resolution usually center on analysis of specific situations, as in the discussion of international conflicts, or, within a developmental framework, in the study of children's reactions to conflict. These studies are aimed toward an understanding of certain aspects of responses in conflict situations without assessment of hard-to-define cognitive and moral developmental factors. Sternberg and Soriano (1984) demonstrated that people seem to have a preferred and consistent conflict-resolution style. They also tested whether such preferences could be predicted with the use of several personality and individual-difference inventories.

In the present study, which partially replicated and extended Sternberg and Soriano's (1984) study, we looked at preferred styles of conflict resolution. The intent was to go beyond the investigation of present conflictresolution styles, in order to begin to understand what formative influences lead a person to develop such styles. As in Sternberg and Soriano's study, subjects read scenarios of international, organizational, or interpersonal conflict and answered several questions about relative preference of possible modes of resolution. In addition, the subjects answered 20 rating-scale questions about their own general conflict-resolution styles and rated the relative importance of several possible formative influences on their own socialization processes of developing attitudes and styles of conflict resolution.

\section{METHOD}

\section{Subjects}

The subjects were 113 female and 94 male introductory psychology students at Kansas State University, who participated in partial fulfill-

\footnotetext{
The authors thank Robert Sternberg for providing copies of the experimental materials and Mark S. Anspach for comments on the manuscript. Requests for reprints should be sent to Richard J. Harris, Department of Psychology, Bluemont Hall, Kansas State University, Manhattan, KS 66506-5302.
}

ment of a course requirement. They were recruited for an experiment in "Factors in formation and consistency of styles of conflict resolution."

\section{Materials}

The scenario stimuli for this study were obtained from Sternberg and Soriano (1984) and were identical to theirs. They consisted of nine stories (about 350 words each) that presented various conflict situations-three stories each for the personal, organizational, and international levels of conflict. The personal conflicts dealt with (1) what a married couple should do about the wife's elderly mother, who was no longer able to live by herself, (2) what a wife should do about a husband whose extremely busy schedule kept him away from her and the children for large blocks of time, and (3) what a man should do about an ex-wife who was preventing him from seeing his children as often as he was permitted to see them by court order. The organizational conflicts dealt with (1) what one faction of an economics department should do about another faction, with which it was competing for scarce computer time, (2) what a leading wire news service should do about a competing service that was taking away its customers, and (3) what a labor union should do with respect to the company's management, which had just presented a disappointing contract offer. The international conflicts dealt with (1) what one nation should do about another with which it shared a river, but which was using its upstream position to divert water supplies from the first country, (2) what one nation should do about another nation that was drilling for shared oil supplies at a rate that was depriving the first nation of its perceived share of the supply, and (3) what one nation should do about another that was undermining its supremacy as the world's leading supplier of processed rubber.

The same seven suggested modes of conflict resolution (also from Sternberg \& Soriano, 1984) were used for all nine stories, although the specifics were changed to fit each particular situation. These seven modes included physical action, economic action, "wait and see," "accept the situation," "step down," third-party intervention, and "undermine esteem." Each solution was presented in statement form and was evaluated in terms of its desirability as a mode of conflict resolution on a Likert-type scale from 1 (excellent choice) to 10 (poor choice).

Following this task, the subjects rated their own attitudes toward conflict resolution on 20 7-point scales, ranging from strongly agree to strongly disagree. These included items designed to probe a variety of attitudes about conflict resolution (e.g., "I resolve conflicts in a consistent way," "I would characterize myself as nonaggressive," "Walking away from conflicts does not really settle anything").

Next, the subjects were asked to rank each of 20 potentially formative influences on conflict-resolution development on a scale from 1 (not influential at all) to 10 (very influential), in terms of "how influential it has been on your forming the way you resolve conflicts." 
Table 1

Mean Conflict Resolution Ratings

\begin{tabular}{lccc}
\hline \multicolumn{1}{c}{ Resolution Type } & Males & Females & Mean \\
\hline & Organizational & & \\
Accept situation & 3.61 & 3.81 & 3.72 \\
Wait and see & 4.90 & 5.06 & 4.99 \\
Undermine esteem & 5.21 & 4.71 & 4.94 \\
Economic action & 4.08 & 4.08 & 4.08 \\
Step down & 4.23 & 4.08 & 4.15 \\
Physical action & 3.82 & 2.63 & 3.17 \\
Third-party intervention & 3.94 & 4.07 & 4.01 \\
Total & 4.26 & 4.06 & 4.15 \\
& International & & \\
Accept situation & 5.09 & 5.21 & 5.15 \\
Wait and see & 5.10 & 5.07 & 5.08 \\
Undermine esteem & 5.08 & 4.90 & 4.98 \\
Economic action & 4.59 & 4.76 & 4.68 \\
Step down & 4.33 & 3.81 & 4.05 \\
Physical action & 4.44 & 3.47 & 3.91 \\
Third-party intervention & 3.07 & 3.08 & 3.07 \\
Total & 4.53 & 4.33 & 4.42 \\
& & & \\
Accept situation & Personal & & \\
Wait and see & 4.89 & 4.56 & 4.71 \\
Undermine esteem & 4.94 & 4.75 & 4.83 \\
Economic action & 5.12 & 2.96 & 3.93 \\
Step down & 5.20 & 3.98 & 4.53 \\
Physical action & 3.91 & 3.75 & 3.82 \\
Third-party intervention & 3.89 & 3.03 & 3.42 \\
Total & 3.20 & 2.54 & 2.84 \\
& 4.45 & 3.65 & 4.01 \\
Accept situation & Overall & & \\
Wait and see & 4.53 & 4.53 & 4.53 \\
Undermine esteem & 4.98 & 4.96 & 4.97 \\
Economic action & 5.14 & 4.19 & 4.63 \\
Step down & 4.63 & 4.27 & 4.43 \\
Physical action & 4.15 & 3.88 & 4.00 \\
Third-party intervention & 3.05 & 3.04 & 3.50 \\
Total & 3.40 & 3.23 & 3.31 \\
\hline Note-1 excellent choce, & 4.41 & 4.01 & 4.19 \\
\hline
\end{tabular}

Note-1 = excellent choice, $10=$ poor choice.

\section{Procedure}

Each subject received two booklets: one containing the nine stories and the questionnaire of 20 attitude scales, the other containing the answer sheets for responding to those stimuli and a sheet for rating the formative influences. The scenarios were presented in a single random order, with the constraint that each block of three contained one of each type of conflict (personal, organizational, international). The statements about resolution styles were presented in a different random order for each of the nine scenarios, though in a constant order across subjects for a given scenario. Subjects first rated these resolutions, next rated the 20 attitude scales, and finally rated the formative influences.

\section{RESULTS}

Results from the ratings of conflict-resolution styles in response to the nine scenarios will be discussed first, followed by the ratings of the formative influences. Responses on the attitude task were used to partition subjects on individual-difference variables; they will be discussed as appropriate in that context.

\section{Resolution Styles}

Results from the responses to conflict-resolution styles in the scenarios were analyzed with a three-way analysis of variance, with a between-subjects factor of sex and within-subjects factors of conflict and resolution type. All main effects and interactions were significant $(p<.02)$. Means are presented in Table 1.

There was a main effect of $\operatorname{sex}[F(1,191)=5.69$, $M S_{\mathrm{e}}=27.99$ ], with women rating the resolutions overall more favorably than men. There was also a main effect of type of conflict $\left[F(2,382)=14.44, M S_{\mathrm{e}}=3.51\right]$, with resolutions of personal conflicts rated more favorably than resolutions of organizational or international conflicts. This was contrasted with the results of Sternberg and Soriano (1984), who found that resolutions to personal conflicts were rated the least favorable of the three types. There was also a conflict type $\times$ sex interaction $[F(2,382)=$ $\left.11.43, M S_{\mathrm{e}}=3.51\right]$, due to the personal conflict solutions' being rated more favorably by the women than any of the other conflict type $\times$ sex combinations.

There was a main effect of resolution type $[F(6,1146)=$ 47.65, $\left.M S_{\mathrm{e}}=4.45\right]$, and an interaction of this factor with $\operatorname{sex}\left[F(6,1146)=5.61, M S_{\mathrm{e}}=4.45\right]$. The latter effect was due to the fact that, for two of the resolution types, "undermine esteem" and physical action, men and women differed markedly (see Table 1, bottom). Women rated each of these resolution types significantly more favorably than men did, though they did not differ on the other five.

Finally, there was an interaction of conflict type and resolution type $\left[F(12,2292)=15.79, M S_{\mathrm{e}}=2.46\right]$, as well as a three-way interaction $[F(12,2292)=3.94$, $\left.M S_{\mathrm{e}}=2.46\right]$. The two-way interaction was due to a very idiosyncratic pattern of ratings of conflict types $\times$ resolution types (Table 1, last column) and of these two interaction cells $\times$ sex. For example, "accept the situation" and economic action are rated relatively more favorably for organizational conflicts, while physical action, "undermine esteem," and third-party intervention are rated relatively favorably as resolutions for personal conflicts. Since each of the conflict types is based on only three scenarios, generalization must be made very cautiously.

In order to further examine the differences tested above, subjects were divided into groups on the basis of their stated propensity toward accepting violence as a solution, so that we could consider individual subject differences. For this purpose, two questions from the attitude scale were used. First, subjects were divided according to their

Table 2

Mean Conflict Resolution Ratings by Conflict Type and Violence-Proneness

\begin{tabular}{lcc}
\hline Conflict Type & Violence-Prone & Non-Violence-Prone \\
\hline Organizational & 4.21 & 4.12 \\
International & 4.38 & 4.47 \\
Personal & 4.38 & 3.76 \\
\hline
\end{tabular}

Note $-1=$ excellent choice, $10=$ poor choice . 
responses to Question 18 ("I would characterize myself as nonaggressive'). Using an approximate median split, subjects were classified as nonaggressive (1-3 for the agree end of the scale) or aggressive (4-7 for the disagree end of the scale). A four-way analysis of variance of the ratings of the conflict-resolution scenarios was run, using the previous three variables, plus a fourth variable of "aggressiveness." No main effect or interaction involving the aggressiveness factor was significant at the .05 level.

A second four-way analysis of variance was run, this time using the responses to Question 10 ("It is acceptable to use violence to settle conflicts sometimes") to partition the subjects into violence-prone and not violenceprone groups. Overall, the mean responses to this item were 4.96 and 6.12 for male and female subjects, respectively, with 1 being agree and 7 being disagree. Because of this gender difference in the distributions, females responding 7 (58\% of the sample) were considered the nonviolence-prone group. For males, those responding either 6 or 7 (52\% of sample) were included in this group. The four-way analysis of variance including this individualdifference factor produced one significant effect involving that factor, the conflict type $\times$ violence-proneness interaction $\left[F(2,372)=10.60, M S_{\mathrm{e}}=3.35\right]$. As may be seen from the means in Table 2, the violence-prone subjects rated the organizational, international, and personal resolutions about the same, while the non-violence-prone subjects rated the resolutions of the personal conflicts as the best and the resolutions of the international conflicts as the worst. Contrary to what might have been expected, however, the violence-prone and non-violence-prone subjects did not differ in their ratings of the different resolution types.

\section{Formative Influences}

Mean ratings of the formative influences appear in Table 3. For both men and women, parents, friends, family, and the general culture were rated the most influential. Generally, sports and coaches were a higher influence among men than among women, whereas church, pastors/ priests, and counselors were somewhat stronger influences on women than on men. Relatively speaking, television, films, books, and law-enforcement officials were some of the lowest rated influences. However, since most of these means were at or above the midpoint of the scale, their influence cannot be discounted.

To investigate the possibility that certain influences would be more prone to socialize certain kinds of values regarding conflict resolution, the means were recomputed for the $52 \%$ of the males and $58 \%$ of the females classi-
Table 3

Mean Ratings of Formative Influences, Rank-Ordered by Sex

\begin{tabular}{lllll}
\hline \multicolumn{1}{c}{ Men } & & & \multicolumn{2}{c}{ Women } \\
\cline { 5 - 6 } Parents & 8.32 & & Parents & 8.76 \\
Friends & 7.55 & & Family & 8.02 \\
Family & 6.96 & & Friends & 7.81 \\
Culture & 6.63 & & Culture & 7.41 \\
Extracurricular & & & Church & 7.00 \\
$\quad$ activities & 6.38 & & Teachers & 6.88 \\
Teachers & 6.38 & & Classes & 6.69 \\
Neighborhood & 6.26 & & Extracurricular & \\
Sports & 6.21 & & activities & 6.46 \\
Church & 6.10 & & Pastors/priests & 6.21 \\
Classes & 6.00 & & Neighborhood & 6.20 \\
Coaches & 5.96 & & Counselors & 6.09 \\
Books & 5.57 & & Speakers & 6.01 \\
Pastors/priests & 5.53 & & Films & 5.75 \\
Speakers & 5.49 & Law officers & 5.74 \\
Counselors & 5.38 & Coaches & 5.35 \\
Law officers & 5.27 & Books & 5.32 \\
Films & 5.03 & Television & 5.14 \\
Television & 4.63 & Sports & 5.03 \\
\hline N & &
\end{tabular}

Note $-1=$ not influential at all, $10=$ very influential.

fied above as non-violence-prone on the basis of responses to Question 10 on the attitude task. The mean ratings of the 20 influences were almost identical to the overall ratings, with the following exceptions: coaches, sports, and films were rated as less influential by both sexes, and teachers were rated as more influential by males.

\section{DISCUSSION}

In the present study, we failed to replicate Sternberg and Soriano (1984) with regard to the preferred resolution of the different types of conflicts. A closer look at their study may suggest some of the reasons. They used only 32 subjects, but we used 207 . Results from both studies showed highly idiosyncratic responses to several of the scenarios, which were the same in both studies-even ones classified in the same conflict type or resolution type. They may not have been fair representatives of their intended category. Also, the resolutions were often fairly mild examples of a particular category (e.g., physical action was not physical violence); this may have reduced differences of resolution style and made them harder to reliably detect.

Nevertheless, Sternberg and Soriano (1984) offered a viable method of studying conflict resolution, a method that does not deserve the neglect it seems to have received since publication of their study. Finally, the study of individual differences in propensity to use the various types of conflict resolution bears much further work, especially with regard to the question of how these propensities are formed.

\section{REFERENCE}

Sternberg, R. J., \& Soriano, L. J. (1984). Styles of conflict resolution. Journal of Personality \& Social Psychology, 47, 115-126.

(Manuscript received August 3, 1989.) 